\title{
Association of Elevated Urinary miR-126, miR-155, and miR-29b with Diabetic Kidney Disease
}

Cristina Beltrami, ${ }^{*}$ Kate Simpson, ${ }^{* \dagger}$ Mark Jesky ${ }^{\ddagger}$ Alexa Wonnacott, ${ }^{*}$ Christopher Carrington, ${ }^{*}$ Peter Holmans, ${ }^{\S}$ Lucy Newbury, ${ }^{*}$ Robert Jenkins, ${ }^{*}$ Thomas Ashdown, ${ }^{*}$ Colin Dayan, ${ }^{\top}$ Simon Satchell, Peter Corish, ${ }^{* *}$ Paul Cockwell, ${ }^{\ddagger}$ Donald Fraser, ${ }^{* \dagger}$ and Timothy Bowen* ${ }^{* \dagger}$

\begin{abstract}
From the Wales Kidney Research Unit,* Division of Infection and Immunity, the MRC Centre for Neuropsychiatric Genetics and Genomics, ${ }^{\S}$ Division of Psychological Medicine and Clinical Neurosciences, and the Diabetes Research Group, "Division of Infection and Immunity, School of Medicine, College of Biomedical and Life Sciences, and the Cardiff Institute of Tissue Engineering and Repair, ${ }^{\dagger}$ Cardiff University, Cardiff; the Department of Renal Medicine, ${ }^{\ddagger}$ Queen Elizabeth Hospital Birmingham, Birmingham; Bristol Renal, ${ }^{\|}$Bristol Medical School, University of Bristol, Bristol; and the BBI Group, ** The Courtyard, Cardiff, United Kingdom
\end{abstract}

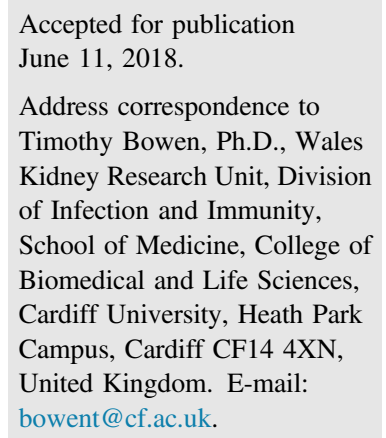

\begin{abstract}
Effective diabetic kidney disease (DKD) biomarkers remain elusive, and urinary miRNAs represent a potential source of novel noninvasive disease sentinels. We profiled 754 miRNAs in pooled urine samples from DKD patients $(n=20)$, detecting significantly increased miR-126, miR-155, and miR-29b compared with controls $(n=20)$. These results were confirmed in an independent cohort of 89 DKD patients, 62 diabetic patients without DKD, and 41 controls: miR-126 (2.8-fold increase; $P<0.0001)$, miR-155 (1.8-fold increase; $P<0.001)$, and miR-29b (4.6-fold increase; $P=0.024)$. Combined receiver operating characteristic curve analysis resulted in an area under the curve of 0.8 . A relative quantification threshold equivalent to $80 \%$ sensitivity for each miRNA gave a positive signal for $48 \%$ of DKD patients compared with $3.6 \%$ of diabetic patients without DKD. Laser-capture microdissection of renal biopsy specimens, followed by quantitative RT-PCR, detected miR-155 in glomeruli and proximal and distal tubules, whereas miR-126 and miR-29b were most abundant in glomerular extracts. Subsequent experiments showed miR-126 and miR-29b enrichment in glomerular endothelial cells (GEnCs) compared with podocytes, proximal tubular epithelial cells, and fibroblasts. Significantly increased miR126 and miR-29b were detected in GEnC conditioned medium in response to tumor necrosis factor- $\alpha$ and transforming growth factor- $\beta 1$, respectively. Our data reveal an altered urinary miRNA profile associated with DKD and link these variations to miRNA release from GEnCs. (Am J Pathol 2018, 188: 1982-1992; https://doi.org/10.1016/j.ajpath.2018.06.006)
\end{abstract}

Recent estimates suggest that 1 in 12 of the global population experiences diabetes mellitus, and approximately $40 \%$ of those affected will go on to develop diabetic kidney disease (DKD). ${ }^{1}$ DKD is the leading cause of end-stage renal disease, and predisposing factors include genetic causes, ethnicity, hyperglycemia, insulin resistance, intraglomerular hypertension, and hyperfiltration. ${ }^{2,3}$
Supported by the National Institute for Health Research Invention for Innovation (i4i) Programme grant II-LA-0712-20003 (T.B. is the principal investigator for the grant) and the Kidney Research UK Project grant awards RP44/2014 (T.B.) and IN4/2013 (S.S.). The JABBS Foundation funded collection of the Renal Impairment in Secondary Care cohort (P.Coc.). The Wales Kidney Research Unit is funded by core support from Health and Care Research Wales (D.F.).

D.F. and T.B. contributed equally to this work as senior authors.

Disclosures: C.D. is an advisor for Novo Nordisk and provides services to Sanofi Genzyme and AstraZeneca (2015 to 2017); S.S. received travel support from Boehringer Ingelheim and UCB UK grant support (2013 to 2015); P.Cor. serves as a consultant for Life Sciences Bridging Fund Wales Consultancy (2015 to 2017); P.Coc. received funding from JABBS Foundation (2014 to 2015); T.B. and D.F. are inventors for patent WO/2017/129977 Chronic Kidney Disease Diagnostic.

The views expressed in this article are those of the authors and not necessarily those of the National Health Service, the National Institute for Health Research, or the Department of Health (United Kingdom). 
Hyperglycemia results in numerous deleterious consequences, including up-regulated cytokine synthesis, reninangiotensin system activation, generation of advanced glycation end products and reactive oxygen species, and increased protein kinase $\mathrm{C}$ activity. ${ }^{4,5}$ Nitric oxide and NF$\kappa \mathrm{B}$ pathway-driven loss of endothelial and vascular modulation have been implicated in insulin resistance, and early DKD may be associated with insulin signaling defects specific to the podocyte. ${ }^{6}$ These insults result in loss of glomerular filtration rate and ultimately renal failure from mesangial hyperexpansion, nodular glomerulosclerosis, and tubulointerstitial fibrosis.?

Detection of urinary microalbuminuria currently forms the basis of DKD progression monitoring, varying from normal mean albuminuria values of approximately $10 \mathrm{mg} /$ day to a diagnosis of microalbuminuria at 30 to $300 \mathrm{mg} / \mathrm{day}$ and macroalbuminuria $>300 \mathrm{mg} / \mathrm{day}^{8}{ }^{8}$ Prognosis is complicated, because not all microalbuminuric patients progress to overt nephropathy. Several novel biomarkers have been assessed for utility in DKD, but none are being used as routine clinical markers, and they may lack specificity and sensitivity to predict individual DKD patient outcomes. In light of the above, novel markers that can discriminate etiology, progression, and/or response to treatment remain highly desirable.

miRNAs are ubiquitously expressed short noncoding RNAs that regulate the expression of most protein coding genes in the human genome, and detection of miR-192, miR-194, miR-215, miR-216, miR-146a, miR-204, and miR-886 is elevated in the kidney. ${ }^{9}$ Urinary miRNAs represent a highly promising novel source of noninvasive biomarkers that are stabilized via argonaute 2 protein/ exosome association and are rapidly and precisely detected by quantitative RT-PCR (RT-qPCR). ${ }^{10}$

Reports have suggested a role for miRNAs in the pathology of DKD, ${ }^{11,12}$ including previous work from this laboratory showing decreased miR-192 in biopsy specimens from late-stage DKD patients with diminished renal function. ${ }^{13}$ However, comparatively little is known about the abundance of urinary miRNAs in DKD patients.

We hypothesized that alterations in urinary miRNA profiles would be associated with DKD. Candidate DKD biomarkers were identified by comparing miRNA profiles in urine samples from a patient discovery cohort with those from unaffected controls. Selected candidates were then measured in a larger independent cohort. Subsequently, laser-capture microdissection (LCM) of renal biopsy specimens and in vitro cell culture were used to investigate the sources of our candidate urinary miRNA DKD biomarkers with respect to nephron domain and cell type.

\section{Materials and Methods}

\section{Study Participants}

DKD was defined in accordance with the National Kidney Foundation Kidney Disease Outcomes Quality Initiative Clinical Practice Guidelines and Clinical Practice Recommendations for Diabetes and Chronic Kidney Disease (CKD). ${ }^{14}$ Accordingly, CKD should be attributable to diabetes in the presence of macroalbuminuria (in the absence of urinary infection), in the presence of microalbuminuria with concomitant diabetic retinopathy, or in type 1 diabetes of at least 10 years' duration. ${ }^{14}$ The initial profiling study cohort

Table 1 Demographic and Clinical Parameters of Patients Recruited from Two Centers

\begin{tabular}{lll}
\hline & Patients $(n=151)$ & \\
\cline { 2 - 3 } Feature & Diabetic $(n=62)$ & DKD $(n=89)$ \\
\hline Male sex, $n(\%)$ & $37(58)$ & $55(62)$ \\
Nonwhite, $n(\%)$ & $13(21)$ & $33(37)$ \\
Age in years, means \pm SD & $52 \pm 16.1$ & $62 \pm 13.6$ \\
eGFR, mL/minute per $1.73 \mathrm{~m}^{2}$ & & \\
$\quad$ Means \pm SD & $78 \pm 16.3$ & $30 \pm 20.9$ \\
Median (IQR) & $84(72-90)$ & $22(17-38)$ \\
CKD stage, $n(\%)$ & & $2(2)$ \\
No CKD/CKD G1 (eGFR $\geq 90 \mathrm{~mL} / \mathrm{minute}$ per $\left.1.73 \mathrm{~m}^{2}\right)$ & $23(37)$ & $10(11)$ \\
CKD G2 (eGFR $=60-89 \mathrm{~mL} / \mathrm{minute}$ per $\left.1.73 \mathrm{~m}^{2}\right)$ & $32(52)$ & $17(19)$ \\
CKD G3 (eGFR $=30-59 \mathrm{~mL} / \mathrm{minute}$ per $\left.1.73 \mathrm{~m}^{2}\right)$ & $5(8)$ & $45(51)$ \\
CKD G4 (eGFR $=15-29 \mathrm{~mL} / \mathrm{minute}$ per $\left.1.73 \mathrm{~m}^{2}\right)$ & $2(3)$ & $15(17)$ \\
CKD G5 (eGFR $<15 \mathrm{~mL} / \mathrm{minute}$ per $\left.1.73 \mathrm{~m})^{2}\right)$ & 0 & \\
ACR, $n(\%) *$ & & $15(16.9)$ \\
A1: normal-high normal $(\mathrm{ACR}<3 \mathrm{mg} / \mathrm{mmol})$ & $54(87.1)$ & $25(28.1)$ \\
A2: moderately increased $\left(\mathrm{ACR}=3-30 \mathrm{mg} / \mathrm{mmol}^{*}\right)$ & $8(12.9)$ & $49(55.0)$ \\
A3: severely increased $(\mathrm{ACR}>30 \mathrm{mg} / \mathrm{mmol})$ & $0(0)$ & \\
\hline
\end{tabular}

The two UK centers were as follows: Wales Kidney Research Tissue Bank, Cardiff (University Hospital Wales) and Birmingham (University Hospital Birmingham, Renal Impairment in Secondary Care Study Cohort).

${ }^{*} \mathrm{ACR}$ group cutoffs and nomenclature derived from Kidney Disease: Improving Global Outcomes 2012 recommendations. ${ }^{14}$

ACR, albumin/creatinine ratio; CKD, chronic kidney disease; DKD, diabetic kidney disease; eGFR, estimated glomerular filtration rate; IQR, interquartile range. 
of 20 DKD patients and 20 healthy controls was obtained from the Wales Kidney Research Tissue Bank, University Hospital of Wales (Cardiff, UK). The DKD group was predominantly male $(85 \%)$, and their mean age was 72 years (SD, \pm 8.7 years). DKD patients were CKD stage 3 to 5 (predialysis), with a mean estimated glomerular filtration rate (eGFR) of $29 \mathrm{~mL} /$ minute per $1.73 \mathrm{~m}^{2}$ (SD, $\pm 8.5 \mathrm{~mL} /$ minute per $1.73 \mathrm{~m}^{2}$ ) and a mean urinary albumin/creatinine ratio (ACR) of $13.5 \mathrm{mg} / \mathrm{mmol}(\mathrm{SD}, \pm 14.5 \mathrm{mg} / \mathrm{mmol})$. The control group $(n=20)$ in the profiling cohort was $50 \%$ male, and their mean age was 47 years (SD, \pm 11.0 years); they had no microalbuminuria (ACR, $<3 \mathrm{mg} / \mathrm{mmol}$ ). For further details on ACR categories, see Table 1.

The confirmation cohort was drawn from two secondary care facilities: the Wales Kidney Research Tissue Bank (as above) and the Renal Impairment in Secondary Care study, University Hospital of Birmingham (Birmingham, UK). ${ }^{15}$ Eighty-nine patients with DKD, including three patients with type 1 diabetes, and 41 healthy controls were recruited across the two sites. An additional control group of 62 diabetic patients without DKD were recruited from Cardiff, including 17 patients with type 1 diabetes. Ethical approval was granted by the Wales Kidney Research Tissue Bank Governance Committee and the South Birmingham Local Research Ethics Committee, respectively.

Patient demographics and clinical parameters are shown in Table 1. All patients were recruited from specialist nephrology and diabetes care services at the two sites during the period spanning autumn 2010 to autumn 2013. DKD patients from the Renal Impairment in Secondary Care study cohort were predominantly advanced nephropaths, as per Renal Impairment in Secondary Care protocol inclusion criteria: briefly, patients with CKD stages 4 to 5 (predialysis) or CKD stage 3 and accelerated progression and/or proteinuria, as defined by the UK National Institute for Health and Care Excellence 2008 CKD guideline for secondary care review. The diabetic patient control group all had a diagnosis of diabetes by standard American Diabetes Association criteria, ${ }^{16}$ but without evidence of DKD (ie, not fulfilling the Kidney Disease Outcomes Quality Initiative criteria).

At the initial clinic visit, renal function was recorded using eGFR, calculated using the modification of diet in renal disease equation. ${ }^{17}$ Urine samples were aliquoted for ACR assessment and for RNA extraction (see below). ACR cutoffs for disease severity were defined as per Kidney Disease: Improving Global Outcomes 2012 guidelines. ${ }^{18}$

\section{Urine Collection, RNA Isolation, and RT-qPCR Analysis}

Urine samples were collected, and RNA extraction from 350 $\mu \mathrm{L}$ of urine, generation of cDNA from equal volumes of RNA extracts, and RT-qPCR were then performed, as described in detail elsewhere. ${ }^{10}$ TaqMan assays (Thermo Fisher Scientific, Paisley, UK) used in this study were as follows: hsa-miR-29b-3p (identification number 000413), hsa-miR-126-3p (identification number 002228), hsa-miR- 155-5p (identification number 002623), and hsa-miR-191$5 p$ (identification number 002299). Relative quantities were calculated using the $2^{-\Delta \Delta \mathrm{Ct}}$ method, and miRNA expression was normalized to hsa-miR-191-5p. ${ }^{10}$

\section{miRNA Profiling by TaqManArray Human MicroRNA Cards}

Urinary miRNAs were reverse transcribed using the Megaplex Primer Pools (Human Pools A version 2.1 and B version 3.0; Thermo Fisher Scientific) with a predefined pool of 381 reverse transcription primers for each Megaplex Primer Pool. A fixed volume of $3 \mu \mathrm{L}$ of RNA solution was used as input in each reverse transcription reaction, and reverse transcription reactions were performed according to the manufacturer's recommendations. Reverse transcription reaction products were amplified using Megaplex PreAmp Primers (Primers A version 2.1 and B version 3.0; Thermo Fisher Scientific), the samples were then diluted to a final volume of $100 \mu \mathrm{L}$, and control subject and DKD patient products were pooled as follows.

To exclude the possibility that sex, age, and eGFR status had extreme effects on miRNA expression profiles, the following pooling strategy was followed: control pool 1, urine samples from 5 females of average age 44.8 years; control pool 2, 5 females, with average age of 57.6 years; control pool 3, 5 males, with average age of 35.2 years; and control pool 4, 5 males, with average of 53.2 years. The following patient pool (PPs) were used: PP1, urine samples from five CKD3 patients with an eGFR between 43.3 and $36 \mathrm{~mL} /$ minute per $1.73 \mathrm{~m}^{2}$; PP2, five stage 3 patients, with an eGFR from 35 to $31 \mathrm{~mL} /$ minute per $1.73 \mathrm{~m}^{2}$; PP3, five stage $4 / 5$ patients, with an eGFR from 27.3 to $23 \mathrm{~mL} /$ minute per $1.73 \mathrm{~m}^{2}$; and PP4, five stage $4 / 5$ patients, with eGFR from 22 to $12.9 \mathrm{~mL} /$ minute per $1.73 \mathrm{~m}^{2}$.

TaqManArray Human MicroRNA Cards A version 2.1 and B version 3.0 (Thermo Fisher Scientific) were used to quantify 754 human miRNAs. Each array included 377 test miRNAs, three endogenous controls, and a negative control. Quantitative PCR was performed on an Applied Biosystems 7900HT thermocycler (Thermo Fisher Scientific) using the manufacturer's recommended program.

\section{LCM from Renal Biopsy Samples}

Glomeruli and proximal tubular and distal tubular profiles were microdissected from sections ( $6 \mu \mathrm{m}$ thick) obtained from five formalin-fixed, paraffin-embedded archived renal biopsy samples from unaffected individuals using the Arcturus Pixcell IIe infrared laser enabled LCM system (Thermo Fisher Scientific).

\section{Cell Culture}

Human conditionally immortalized glomerular endothelial cell (GEnC) and human podocyte cell lines were propagated 
at $33^{\circ} \mathrm{C}$, as described previously. ${ }^{19,20}$ After 5 (GEnC) and 14 (podocyte) days, cells were transferred to $37^{\circ} \mathrm{C}$ incubation to inactivate the SV40 T antigen and permit differentiation, before experimental use. Where stated, GEnCs were growth arrested for 24 hours and then treated with $10 \mathrm{ng} / \mathrm{mL}$ tumor necrosis factor (TNF)- $\alpha$ or $1 \mathrm{ng} / \mathrm{mL}$ transforming growth factor (TGF)- $\beta 1$ at either $5 \mathrm{mmol} / \mathrm{L}$ normoglycemic or $25 \mathrm{mmol} / \mathrm{L}$ hyperglycemic D-glucose concentrations for 24 hours. Proximal tubular epithelial cell line $\mathrm{HK}-2^{21}$ and fibroblast $^{22}$ cultures were maintained as described elsewhere. Cells and culture medium obtained from each well were used for RNA extraction, as described above.

\section{Statistical Analysis}

miRNA profiling data were analyzed using Thermo Fisher Scientific's DataAssist Software version 3.01, NormFinder Software version 0.953 (http://moma.dk/normfindersoftware, last accessed February 21, 2018), and GraphPad Prism 6 version 6.0d (GraphPad Software, La Jolla, CA). Pearson correlation coefficients were used to detect clusters of similarity in miRNA threshold cycle values between each pool group in patients and between each pool group in controls. To identify a suitable reference gene for the normalization of miRNA expression in this study, the NormFinder algorithm was applied to the expression data obtained from the Human TaqMan miRNA Arrays. Analysis comparing miRNA levels between subjects with DKD and controls was performed using GraphPad Prism 6 version 6.0d. $P<0.05$ was considered statistically significant. miRNA profiling data sets can be found in Gene Expression Omnibus (https:// www.ncbi.nlm.nih.gov/geo; accession number GSE114477).

\section{Results}

\section{Altered Urinary miRNA Detection in DKD Patients}

To select candidate miRNAs that may act as DKD biomarkers, data from unbiased expression profiling of 754 miRNAs in urine samples from 20 DKD patients and 20 unaffected controls were compared. Analyses were performed on four patient and four control pools, each composed of urine samples from five individuals, as recommended by Zhang and Gant. ${ }^{23}$ Samples were pooled before profiling to minimize the contribution of subject-tosubject variation and to make substantive features easier to find, and thereby identify biomarkers common across individuals. $^{24}$ Previous analysis suggested that 40 individuals might optimally be pooled across eight arrays, ${ }^{23}$ which was our chosen pooling approach.

miRNAs for which statistically significant fold-change increases were detected in patient urine compared with control samples (12 data points) and the corresponding down-regulated miRNAs (35 data points) are shown in Figure 1A. The fold-change data for these 47 miRNAs are summarized in Figure 1B, and the 8 miRNAs exhibiting greater than fivefold change were subsequently selected as potential candidate biomarkers for further analysis.

Specific RT-qPCR assays were then used to analyze these miRNAs in each component urine sample pooled for profiling analysis. Statistically significant differences in miRNA detection between DKD patient and control urine samples were replicated for miR-126 (4.3-fold increase; $P=0.0087$ ), miR-155 (22.9-fold increase; $P=0.0024$ ), and miR-29b (4.9-fold increase; $P=0.0002$ ) (Figure 1, C-E).

\section{Elevated Urinary miR-126, miR-155, and miR-29b Detection in an Independent DKD Patient Cohort}

To test the above findings, miR-126, miR-155, and miR$29 \mathrm{~b}$ were quantified in samples from an independent cohort of patients with established DKD from the Renal Impairment in Secondary Care study. ${ }^{15}$ Samples from 89 patients meeting the criteria established in the UK National Institute for Health and Care Excellence 2008 criteria were available. An additional cohort of 62 patients with diabetes mellitus, but without proteinuria or other evidence of DKD, were included, as were samples from 41 individuals without evidence of diabetes or DKD (Table 1). Diabetes patients without DKD were included as a third group in this analysis to identify DKD-specific miRNA detection changes and not purely hyperglycemia-driven effects from our profiling comparison of DKD patients with control individuals.

Significant differences were again seen between DKD patients and controls for miR-126 (2.8-fold increase; $P<0.0001)$ (Figure 2A), miR-155 (1.8-fold increase; $P<0.001$ ) (Figure 2B), and miR-29b (4.6-fold increase; $P=0.024$ ) (Figure 2C). Comparison of DKD patients with diabetic patients without DKD was statistically significant for miR-126 (3.1-fold increase; $P<0.0001$ ) and miR-155 (1.6-fold increase; $P=0.024$ ), with a trend to increased miR-29b (4.1-fold increase; $P=0.121$ ) (Figure 2, A-C).

RT-qPCR data for all three miRNAs were used to compare DKD patients and diabetic patients without DKD in the combined receiver operating characteristic curve analysis shown in Figure 2D, giving an area under the curve of 0.80 . To analyze the contributions of each miRNA to the above receiver operating characteristic curve, individual specificity and likelihood ratios were calculated for relative expression values equivalent to a sensitivity of $80 \% .^{26,27}$ Data displayed in Table 2 illustrate the magnitude of corresponding specificity values was miR-126 $>$ miR$155>$ miR-29b, and that combined miRNA data resulted in a $\geq 6.5 \%$ increase in specificity and likelihood ratio compared with individual miRNAs. These relative expression data were then used as consecutive threshold values to discriminate between DKD and diabetic patients without DKD (Table 3) from the independent cohort. The discriminatory order was miR-29b (DKD/diabetic patients without $\mathrm{DKD}=5.62)>\mathrm{miR}-126(\mathrm{DKD} /$ diabetic patients without 


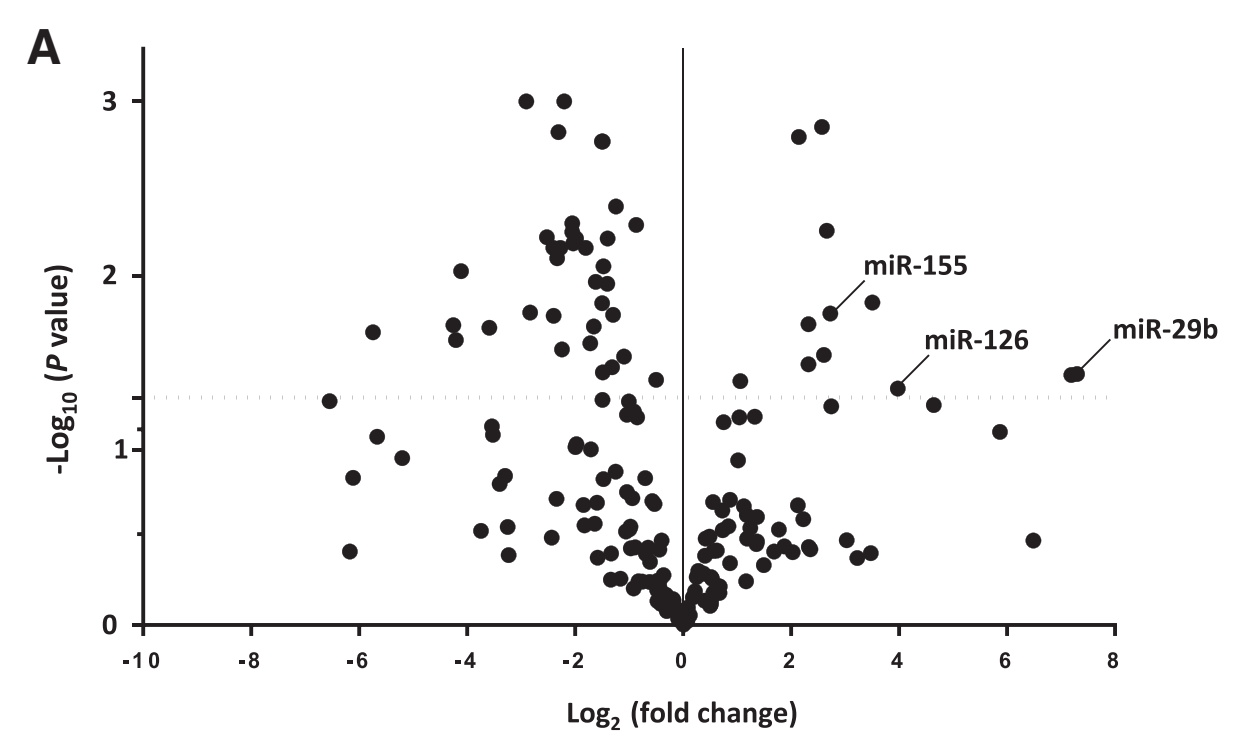

\section{B}

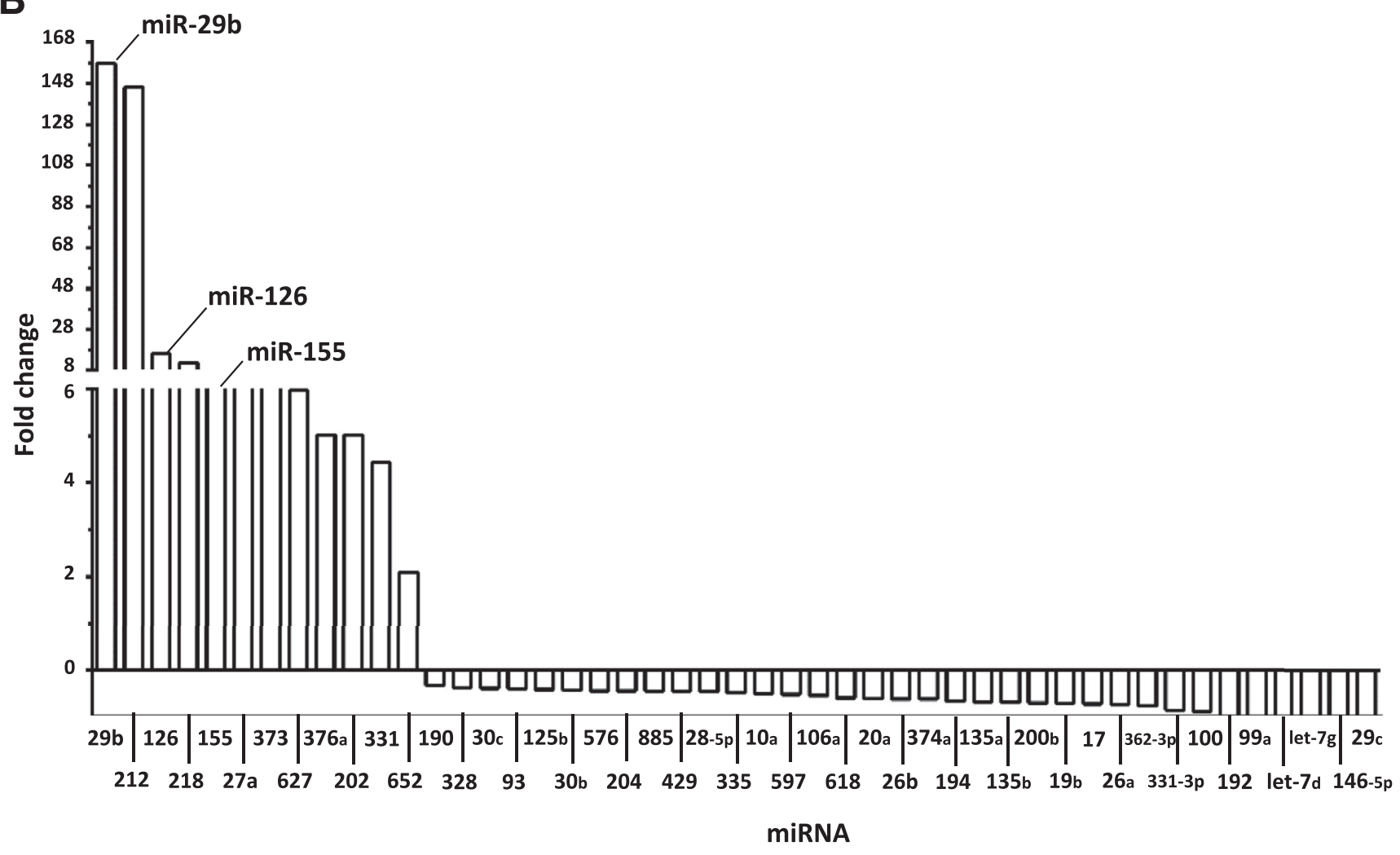

C
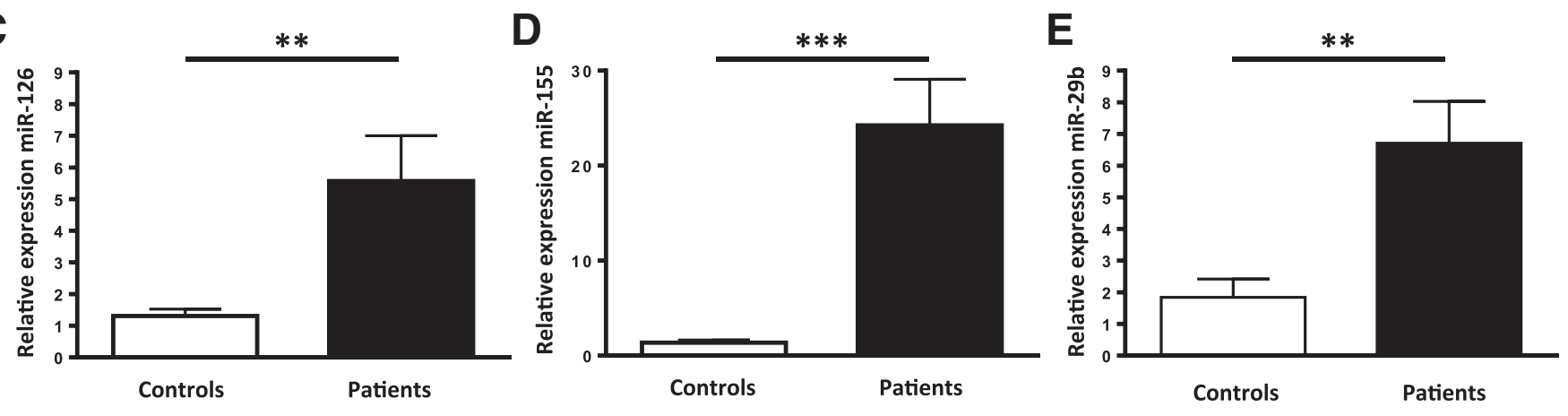
$\mathrm{DKD}=3.48)>\operatorname{miR}-155(\mathrm{DKD} /$ diabetic patients without $\mathrm{DKD}=2.23$ ), and relative expression values exceeding all three thresholds were obtained for $48.0 \%$ of DKD patients compared with $3.6 \%$ of diabetic patients without DKD (Table 3).

\section{LCM Shows Increased Glomerular Abundance of miR- 126 and miR-29b That Is Replicated in GEnC Culture}

Previous reports have linked changes in miRNA expression to DKD pathology, but have focused on whole tissue studies. For example, association of decreased miR-192 expression with disease progression in DKD biopsy specimens by in situ hybridization has been reported. ${ }^{13}$

Herein, LCM was used to isolate glomeruli and proximal and distal tubules (Figure 3A) from histologically normal formalin-fixed, paraffin-embedded renal biopsy samples, and miR-126, miR-155, and miR-29b expression was analyzed by RT-qPCR. In Figure 3B, a typical CD10-stained formalinfixed, paraffin-embedded biopsy section is seen before and after LCM to isolate glomeruli and proximal and distal renal tubules. miR-126, miR-155, and miR-29b were detected in extracts from all three nephron regions (Figure 3, C-E). Increased glomerular abundances were observed for miR-126 (Figure 3C) and miR-29b (Figure 3E), whereas miR-155 was most abundant in the distal tubule (Figure 3D).

Conclusions regarding nephron region-specific miRNA expression from the above analyses are inherently limited, however, because tissue extracts are subject to trace contamination by cells from other nephron domains. Therefore, cellular miRNA localization within each nephron region was subsequently investigated by RT-qPCR analysis of podocyte and endothelial cell (GEnC) cultures from the glomerulus, renal proximal tubular epithelial cells, and fibroblasts. Detection of miR-126 was significantly higher in GEnCs compared with other cell types (Figure 3F). Most miR-155 was detected in proximal tubular epithelial cells and least miR-155 was detected in GEnCs (Figure 3G), whereas miR-29b was most abundant in GEnCs (Figure 3H).

\section{GEnC Release of miR-126 and miR-29b in an in Vitro Model of Hyperglycemia Is Driven by TNF- $\alpha$ and TGF- $\beta 1$, Respectively}

The above data localized the majority of miR-126 and miR$29 \mathrm{~b}$ expression to the GEnC. Stimuli by which miRNAs are released into the glomerular ultrafiltrate, and hence the urine, were investigated next. Data from animal models of diabetes show increased glomerular and proximal tubular epithelial cell TNF- $\alpha$ expression, and renoprotective effects of TGF- $\beta$ inhibitors have also been reported. ${ }^{28,29}$ GEnC expression of our candidate miRNAs was thus analyzed in vitro in response to TNF- $\alpha$ and TGF- $\beta 1$ in normoglycemia and hyperglycemia (Figure 4).

The presence of TNF- $\alpha$ led to significantly increased miR-126 detection in GEnC conditioned medium at 5 and $25 \mathrm{mmol} / \mathrm{L}$ D-glucose (Figure 4B), a pattern also seen for miR-29b after TGF- $\beta 1$ addition (Figure 4D). These cytokines did not increase GEnC expression of miR-126 (Figure 4A) or miR-29b (Figure 4C), a pattern consistent with increased release, but not expression, of miRNAs.

No significant changes in miR-155 were detected in response to elevated D-glucose with either cytokine, and data for TNF- $\alpha$ are shown (Figure 4, E and F). Similarly, changes in miR-126 after TGF- $\beta 1$ addition, and for miR-29b in the presence of TNF- $\alpha$, were not observed (data not shown). Elevated D-glucose alone did not change miRNA expression in GEnCs or conditioned medium (Figure 4).

\section{Discussion}

DKD is the leading cause of kidney failure, requiring renal replacement therapy worldwide, but effective methods to identify and halt progression of disease-specific pathophysiological changes remain elusive. Current effective interventions, such as control of blood glucose and blood pressure, are challenging to achieve, costly, and time intensive. Existing tests track DKD from diabetic diagnosis to kidney failure, but do not allow accurate prognosis for the individual patient. In addition, the absence of treatment response biomarkers hinders development of emerging DKD therapies. There is, thus, an unmet need for additional DKD biomarkers to target intervention and follow response to therapy.

In this study, urinary miRNA DKD biomarkers were identified. Increased detection of miR-126, miR-155, and miR-29b was observed in the urine of DKD patients in comparison with both unaffected individuals and diabetic patients without DKD. miRNA localization and release studies further suggested specific release of miR-126 and miR-29b from GEnCs. This raised the possibility that

\footnotetext{
Figure 1 Urinary miRNA detection in urine samples from diabetic kidney disease (DKD) patients and control subjects. A: Volcano plot showing the detection profile of the 377 urinary miRNAs in TaqManArray Human MicroRNA Card A in DKD patients and controls. The dotted horizontal line represents a $P$ value boundary of 0.05 . B: Fold change of miRNA detection between DKD patients and controls. DataAssist Softwarewas used to perform relative quantification for sample comparison, to perform $t$-test sample group comparisons, and to produce the graphic output shown. C-E: Quantitative RT-PCR (RT-qPCR) analysis shows significant differences in detection of miR-126 (C), miR-155 (D), and miR-29b (E) between patients and control urine in the component urine samples pooled for profiling analyses (A and B). C-E: DKD patients versus controls for miR-126 (C), miR-155 (D), and miR-29b (E). Analysis was performed by unpaired two-tailed $t$-test with Welch's correction. Profiling data analysis using the NormFinder algorithm identified miR-191 as optimal for normalization of RT-qPCR data. Data were normalized to endogenous control miR-191 and are expressed as means \pm SEM (C-E). $n=20$ (A, DKD patients and controls, four pools of five patients, and $\mathbf{C}-\mathbf{E}$, each group). ${ }^{* *} P<0.01,{ }^{* * *} P<0.001$.
} 

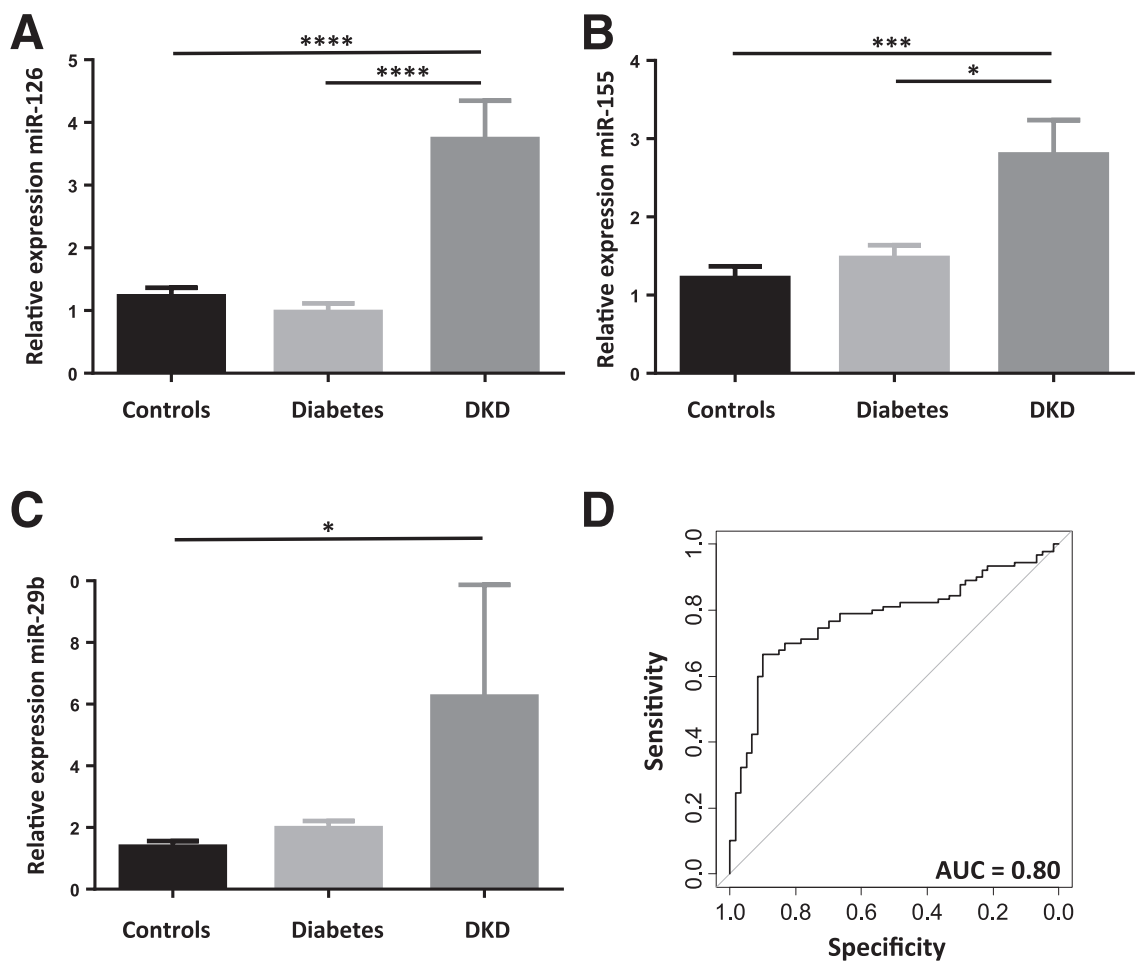

Figure 2 Quantitative RT-PCR detection of selected miRNAs in patients and control subjects. A-C: Relative expression was significantly different in 89 diabetic kidney disease (DKD) patients compared with 62 diabetic patients without DKD and 41 controls for miR-126 (A) and miR-155 (B), and significantly different in DKD patients compared with controls for miR-29b (C). A: DKD patients versus diabetic patients without DKD and controls. B: DKD patients and diabetic patients without DKD; DKD patients versus controls. C: DKD patients versus controls. Analysis was performed by unpaired one-tailed $t$-test with Welch's correction. Data were normalized to endogenous control miR-191. D: Combined receiver operating characteristic curve analysis for miR-126, miR-155, and miR-29b, with an area under the curve (AUC) of 0.80 . Data were generated using the pROC package (http://expasy.org/tools/pROC) ${ }^{25}$ in R-3.2.3. Data are expressed as means \pm SEM (A-C). $n=89$ (A-C, DKD patients); $n=62$ (A-C, diabetic patients without DKD); $n=41$ (A-C, controls). ${ }^{*} P<0.05,{ }^{* *} P<0.001$, and $* * * * P<0.0001$. urinary miRNA quantification might provide data on ongoing pathologic processes, and so aid patient stratification and measurement of response to therapy.

Urinary miRNA biomarkers have several potential significant advantages over circulating miRNAs for adoption into existing treatment pathways alongside current biomarkers, including speed and cost of noninvasive sample access. ${ }^{30}$ However, few urinary miRNA DKD biomarker data have so far been reported. Previous studies have focused on circulating miRNAs and have generated conflicting data with respect to association of miR-126 with diabetes mellitus and/or DKD. A recent cross-sectional analysis of type 2 diabetes mellitus patients found a negative association with plasma miR- $126,{ }^{31}$ and similar findings have been reported for type 1 diabetes mellitus and all complications. ${ }^{32}$ By contrast, miR-126 detection did not change in whole blood from type 2 diabetes mellitus patients and control subjects, but decreased in DKD patient samples. ${ }^{33}$ Furthermore, no change in plasma miR-126 was observed in a study of pediatric type 1 diabetic patients. ${ }^{34}$ These analyses provide inconsistent data for the biomarker utility of circulating miR-126, in contrast to the significant and reproducible increases detected in miR-126, miR-155, and miR-29b in DKD patient urine in the present study.

The DKD-specific alterations in urinary miRNA profiles detected in this study may have functional significance. In vitro analyses localized miR-126 and miR-29b principally to the GEnC, with miR-155 expression distributed evenly across the nephron. Glomerular endothelial localization of miR-126 may reflect the role of this transcript in vascular regulation. Targeted mouse miR-126 deletion resulted in vascular abnormalities by removing inhibition of sprouty-related EVH1 domain-containing protein 1 expression, thereby enhancing vascular endothelial growth factor function. ${ }^{35}$ A role in DKD pathology for vascular endothelial growth factor-A signaling between GEnCs and podocytes has been proposed. ${ }^{36}$ In addition, miR-126 repression of vascular cell adhesion molecule 1 expression in human umbilical vein endothelial cells regulates

Table 2 Sensitivity and Specificity Values, Likelihood Ratios, and RQ Thresholds for miR-126, miR-155, miR-29b, and All Three miRNAs $>80 \%$ ROC Curve Sensitivity Threshold

\begin{tabular}{|c|c|c|c|c|}
\hline Group & Sensitivity, \% & Specificity, \% & Likelihood ratio & RQ threshold \\
\hline All three miRNAs & 80.21 & 63.64 & 2.206 & $>1.148$ \\
\hline $\operatorname{miR}-126$ & 80.41 & 57.14 & 1.876 & $>0.6762$ \\
\hline miR-155 & 80.61 & 52.00 & 1.679 & $>0.9110$ \\
\hline miR-29b & 80.61 & 40.00 & 1.344 & $>0.8058$ \\
\hline
\end{tabular}

$R O C$, receiver operating characteristic; $R Q$, relative expression. 
Table 3 DKD and D Patient Numbers and Percentages $>80 \%$ ROC Curve Sensitivity Threshold for miR-126, miR-155, miR-29b, and All Three miRNAs

\begin{tabular}{lcccc}
\hline Group & miR-126 & miR-155 & miR-29b & All three miRNAs \\
\hline Patients $>80 \%$ sensitivity threshold, $n(\%)$ & & & \\
D & $23(41.8)$ & $30(54.6)$ & $13(23.6)$ & $2(3.6)$ \\
DKD & $80(81.6)$ & $67(68.4)$ & $73(74.5)$ & $47(48.0)$ \\
\hline
\end{tabular}

D, diabetic patients without DKD; DKD, diabetic kidney disease; ROC, receiver operating characteristic.

their response to proinflammatory adhesion molecules. ${ }^{37}$ miR-126 has also been implicated in the heterogenic inflammatory response of renal microvascular endothelial cells. $^{38}$

Increased expression of miR-155 has been observed in DKD patient renal biopsy specimens, in close correlation with increased serum creatinine. ${ }^{39}$ Furthermore, miR-155 deficiency attenuated renal damage and IL-17 expression was down-regulated in streptozotocin-induced DKD mice. ${ }^{40}$ Together with miR-126, miR-155 has been implicated in multiple forms of vascular remodeling and associated with cardiovascular disease. $^{41}$
A

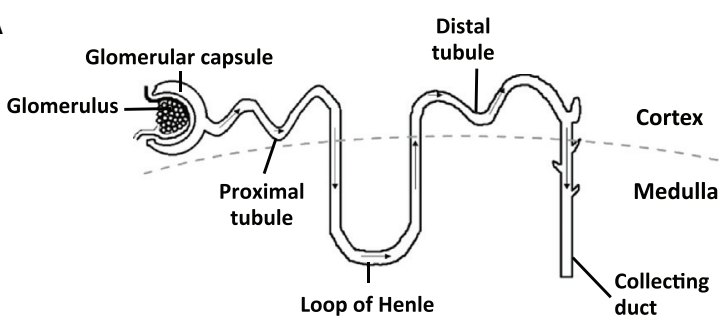

C

LCM-dissected nephron domains

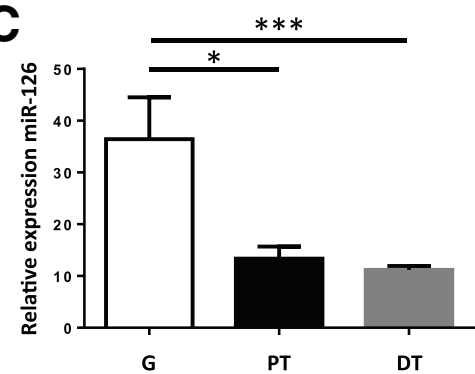

F

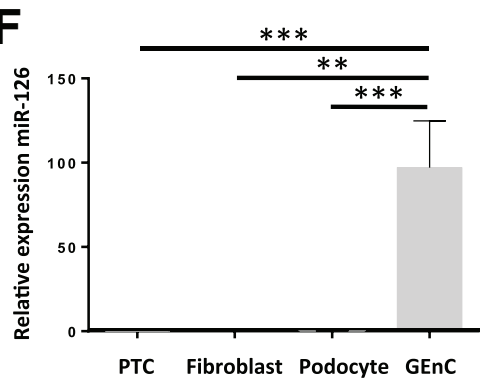

D

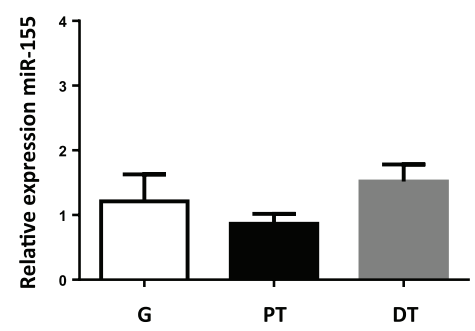

G

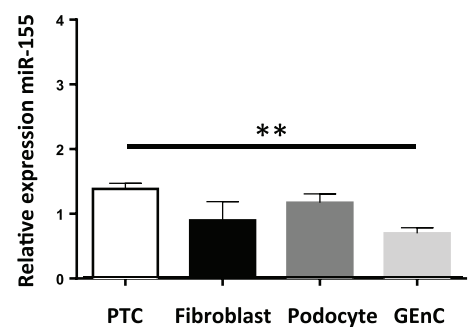

B

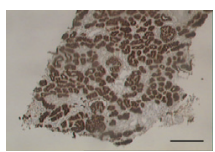

Renal biopsy specimen before LCM

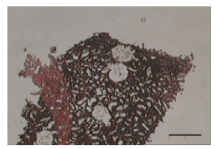

Renal biopsy specimen after LCM

E
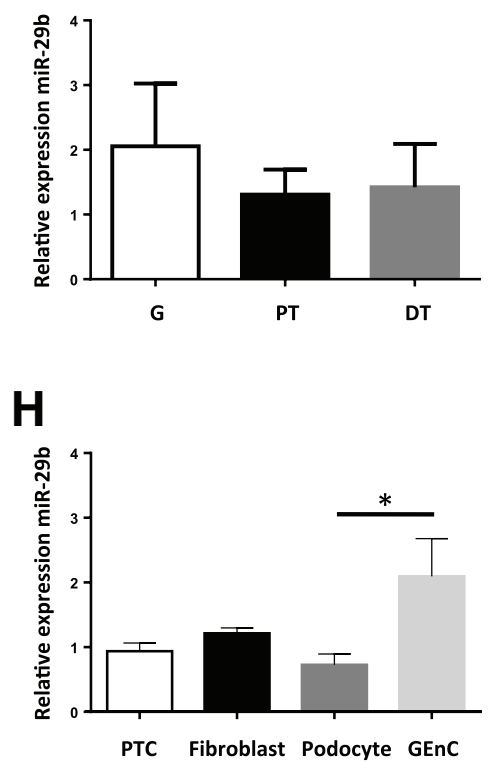

Figure 3 Localization of miRNA expression by laser-capture microdissection (LCM) and cell culture. A: Key functional nephron domains include the glomerulus (G), the proximal tubule (PT), and the distal tubule (DT). B: A CD10-stained formalin-fixed, paraffin-embedded renal biopsy sample before and after excision of glomeruli by LCM. C-E: Relative expression of miR-126, miR-155, and miR-29b, respectively, in LCM-isolated Gs, PTs, and DTs from five renal biopsy specimens of healthy individuals. C: Statistically significant differences in miRNA expression were observed in G versus PT, and G versus DT. F-H: Relative expression of miR-126, miR-155, and miR-29b, respectively, in in vitro cultured HK-2 renal proximal tubular epithelial cells (PTCs), fibroblasts, podocytes, and conditionally immortalized glomerular endothelial cells (GEnCs). F: Statistically significant differences in miRNA expression were observed in GEnCs versus fibroblasts, and GEnCs versus PTCs and podocytes. G: PTCs versus GEnCs. H: GEnCs versus podocytes. Analysis was performed by one-way analysis of variance with Tukey's multiple comparison test. Data were normalized to endogenous control miR-191 and are presented as means \pm SEM (C-H). $n=5$ (C, biopsy specimens); $n=4$ (H). ${ }^{*} P<0.05,{ }^{* *} P<0.01$, and ${ }^{* * *} P<0.001$. Scale bars $=100 \mu \mathrm{m}$ (B). 
A
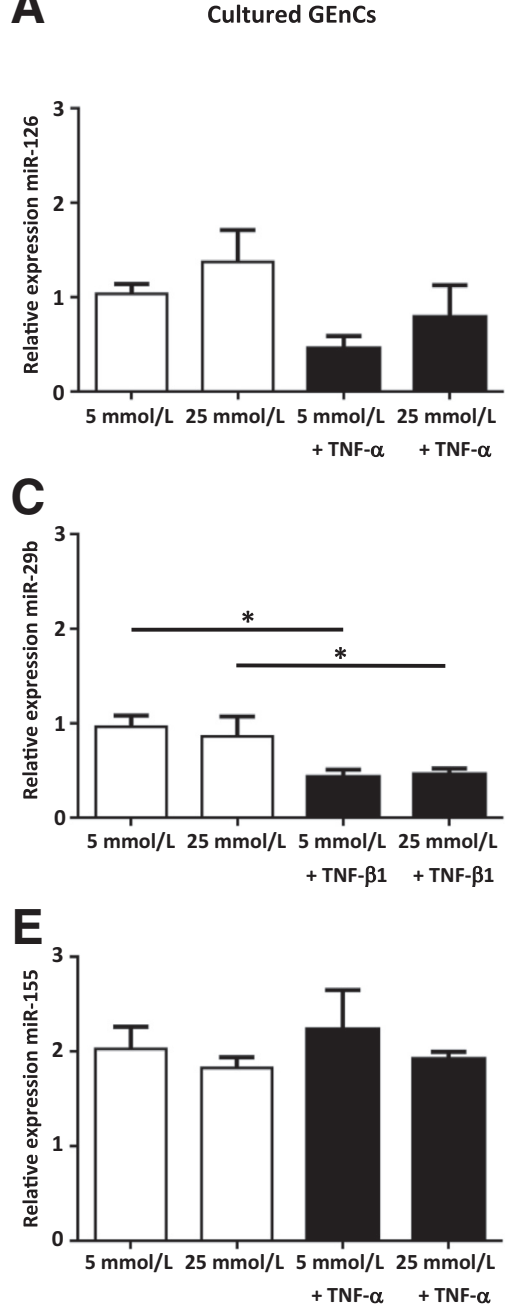
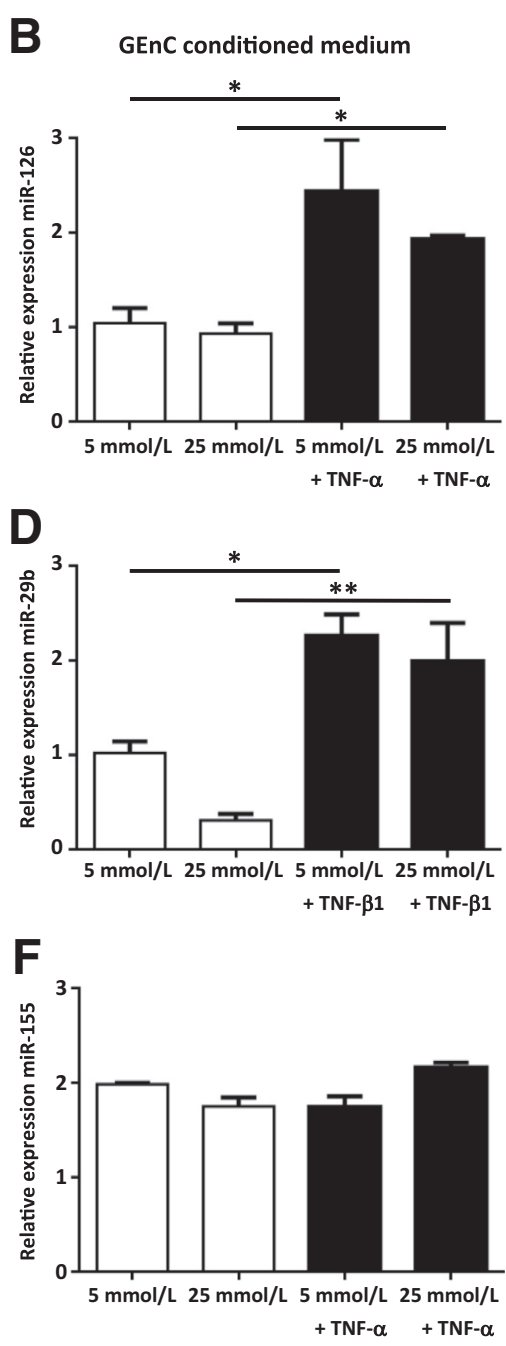

Figure 4 miRNA expression in glomerular endothelial cells (GEnCs) and GEnC conditioned medium in response to hyperglycemia and diabetic kidney disease-related cytokines. After 24 hours of culture in 5 or $25 \mathrm{mmol} / \mathrm{L}$ D-glucose, relative expression in GEnCs and GEnC conditioned medium, respectively, of miR-126 in response to $10 \mathrm{ng} / \mathrm{mL}$ tumor necrosis factor (TNF)- $\alpha$ (A and $\mathbf{B})$, miR$29 \mathrm{~b}$ in response to $1 \mathrm{ng} / \mathrm{mL}$ transforming growth factor (TGF)- $\beta 1$ (C and D), and miR-155 in response to $10 \mathrm{ng} / \mathrm{mL}$ TNF- $\alpha$ (E and $\mathbf{F})$, and in untreated cells. B: Statistically significant differences in miRNA expression were observed in study of $5 \mathrm{mmol} / \mathrm{L} \mathrm{D}$-glucose versus $5 \mathrm{mmol} / \mathrm{L} \mathrm{D}$-glucose plus TNF- $\alpha$, and $25 \mathrm{mmol} / \mathrm{L}$ D-glucose versus $25 \mathrm{mmol} / \mathrm{L}$ D-glucose plus TNF- $\alpha$. C: Study of $5 \mathrm{mmol} / \mathrm{L}$ D-glucose versus $5 \mathrm{mmol} / \mathrm{L}$ D-glucose plus TGF- $\beta 1$, and $25 \mathrm{mmol} / \mathrm{L}$ D-glucose versus $25 \mathrm{mmol} / \mathrm{L}$ D-glucose plus TGF- $\beta 1$. D: Study of $5 \mathrm{mmol} / \mathrm{L}$ D-glucose versus $5 \mathrm{mmol} / \mathrm{L}$ D-glucose plus TGF- $\beta 1$, and $25 \mathrm{mmol} / \mathrm{L} \mathrm{D}$-glucose versus $25 \mathrm{mmol} / \mathrm{L}$ $\mathrm{D}$-glucose plus TGF- $\beta 1$. Analysis was performed by one-way analysis of variance with Tukey's multiple comparison test. Data were normalized to endogenous control miR191 and are presented as means \pm SEM (A-F). $n=4$ (D). ${ }^{*} P<0.05,{ }^{*} * P<0.01$.
Decreased miR-29b has been reported in early and advanced animal models of diabetic renal fibrosis. ${ }^{42}$ Chen and colleagues $^{43}$ found that loss of renal miR-29b in $\mathrm{db} / \mathrm{db}$ mice led to increased albuminuria, TGF- $\beta$-mediated fibrosis, and immune injury, whereas restored miR-29b expression inhibited renal injury. Indeed, although upregulated miRNAs were specifically studied, the importance of down-regulated miRNAs cannot be ignored.

miR-29b localized to the glomerular endothelium in this study. Reduction of collagen and laminin synthesis has been reported after forced miR-29b expression in human corneal endothelial cells. ${ }^{44}$ In apolipoprotein E knockout mice, miR$29 \mathrm{~b}$ induced aortic endothelial permeability in response to a high-fat diet and brought about aortic apoptosis by direct targeting of melatonin receptor $\mathrm{mt} 1 .{ }^{45}$ In addition, upregulated miR-29b expression has been observed in human umbilical vein endothelial cells exposed to hyperglycemia. ${ }^{46}$

The cytokine-driven release from GEnCs observed for miR-126 (TNF- $\alpha$ ) and miR-29b (TGF- $\beta 1$ ) reported herein suggests that these cells may be the principal source of elevated urinary miR-126 and miR-29b detected in DKD.
We speculate that this constitutes evidence for diseaserelated signaling down the nephron that will be interesting to test in future studies. Indeed, there is a reported association of urinary miRNAs with exosomes ${ }^{10}$ and exosomal transport, which might facilitate passage of miRNAs through the nephron, for all three candidate biomarker miRNAs.

Exosome-mediated release of miR-126 from $\mathrm{CD} 34^{+}$peripheral blood mononuclear cells is proangiogenic, and decreased miR-126 was detected in elevated glucose cell culture and diabetic patients. ${ }^{47}$ miR-155 is depleted in urinary exosomes from microalbuminuric type 1 diabetes mellitus patients. ${ }^{48}$ Endogenous miR-29b, spontaneously released from beta cells within exosomes, stimulates TNF- $\alpha$ secretion from spleen cells isolated from diabetes-prone nonobese diabetic mice in vitro. ${ }^{49}$

In summary, we have used unbiased profiling approaches to identify a urinary miRNA signature associated with DKD and have subsequently confirmed increased miR-126, miR155 , and miR-29b in an independent patient cohort. miR126 and miR-29b were identified as enriched in GEnCs, and 
released from these cells in response to DKD-related cytokines. Urinary miR-126, miR-155, and miR-29b are, therefore, promising DKD biomarkers, and the potential pathologic significance of miR-126 and miR-29b release from GEnCs merits further evaluation.

\section{Acknowledgments}

We thank control subjects and patients for the donation of urine samples, including those samples kindly provided by coauthors Dr. Mark D. Jesky and Prof. Paul Cockwell (Queen Elizabeth Hospital Birmingham, Birmingham, UK).

C.B. and K.S. performed experiments, generated and analyzed data, and wrote the manuscript; A.W., C.C., L.N., R.J., and T.A. performed experiments and generated and analyzed data; M.J., P.H., C.D., S.S., P.Cor., and P.Coc. discussed elements of experimental design and/or cohort composition; D.F. and T.B. designed the research; T.B. wrote the manuscript, which was edited by D.F. and then amended and approved by each author.

\section{Supplemental Data}

Supplemental material for this article can be found at https://doi.org/10.1016/j.ajpath.2018.06.006.

\section{References}

1. Jha V, Garcia-Garcia G, Iseki K, Li Z, Naicker S, Plattner B, Saran R, Wang AY, Yang CW: Chronic kidney disease: global dimension and perspectives. Lancet 2013, 382:260-272

2. Cowie CC, Port FK, Wolfe RA, Savage PJ, Moll PP, Hawthorne VM: Disparities in incidence of diabetic end-stage renal disease according to race and type of diabetes. N Engl J Med 1989, 321:1074-1079

3. Regele F, Jelencsics K, Shiffman D, Pare G, McQueen MJ, Mann JF, Oberbauer R: Genome-wide studies to identify risk factors for kidney disease with a focus on patients with diabetes. Nephrol Dial Transplant 2015, 30:26-34

4. Makita Z, Radoff S, Rayfield EJ, Yang Z, Skolnik E, Delaney V, Friedman EA, Cerami A, Viassara H: Advanced glycosylation end products in patients with diabetic nephropathy. N Engl J Med 1991, 325:836-842

5. Schrijvers BF, De Vriese AS, Flyvbjerg A: From hyperglycemia to diabetic kidney disease: the role of metabolic, hemodynamic, intracellular factors and growth factors/cytokines. Endocr Rev 2004, 25 : 971-1010

6. Coward RJ, Saleem MA: Podocytes as a target of insulin. Curr Diabetes Rev 2011, 7:22-27

7. Rodriguez-Iturbe B, Johnson RJ, Herrera-Acosta J: Tubulointerstitial damage and progression of renal failure. Kidney Int Suppl 2005, 99: S82-S86

8. Parving H-H, Persson F, Rossing P: Microalbuminuria: a parameter that has changed diabetes care. Diabetes Res Clin Pract 2015, 107:1-8

9. Landgraf P, Rusu M, Sheridan R, Sewer A, Iovino N, Aravin A, et al: A mammalian microRNA expression atlas based on small microRNA sequencing. Cell 2007, 129:1401-1414

10. Beltrami C, Clayton A, Newbury LJ, Corish C, Jenkins RH, Phillips AO, Fraser DJ, Bowen T: Stabilization of urinary microRNAs by association with exosomes and argonaute 2 protein. Noncoding RNA 2015, 1:151-165
11. Trionfini P, Benigni A, Remuzzi G: MicroRNAs in kidney physiology and disease. Nat Rev Nephrol 2015, 11:23-33

12. Simpson K, Wonnacott A, Fraser DJ, Bowen T: MicroRNAs in diabetic nephropathy: from biomarkers to therapy. Curr Diab Rep 2016, 16:35

13. Krupa A, Jenkins R, Luo DD, Lewis A, Phillips AO, Fraser DJ: Loss of MicroRNA-192 promotes fibrogenesis in diabetic nephropathy. J Am Soc Nephrol 2010, 21:438-447

14. KDOQI clinical practice guideline for diabetes and CKD: 2012 update. Am J Kidney Dis 2012, 60:850-886

15. Stringer S, Sharma P, Dutton M, Jesky M, Ng K, Kaur O, Chapple I, Dietrich T, Ferro C, Cockwell P: The natural history of, and risk factors for, progressive chronic kidney disease (CKD): the Renal Impairment in Secondary Care (RIISC) study: rationale and protocol. BMC Nephrol 2013, 14:95

16. American Diabetes Association: Diagnosis and classification of diabetes mellitus. Diabetes Care 2010, 33 Suppl 1:S62-S69

17. Levey AS, Bosch JP, Lewis JB, Greene T, Rogers N, Roth D; Modification of Diet in Renal Disease Study Group: A more accurate method to estimate glomerular filtration rate from serum creatinine: a new prediction equation. Ann Intern Med 1999, 130:461-470

18. Kidney Disease: Improving Global Outcomes (KDIGO) CKD Work Group (2013):: KDIGO 2012 clinical practice guideline for the evaluation and management of chronic kidney disease. Kidney Int Suppl 2013, 3:S1-S150

19. Satchell SC, Tasman CH, Singh A, Ni L, Geelen J, von Rhuland CJ, O'Hare MJ, Saleem MA, van den Heuvel LP, Mathieson PW: Conditionally immortalized human glomerular endothelial cells expressing fenestration in response to VEGF. Kidney Int 2006, 69:1633-1640

20. Saleem MA, O'Hare MJ, Reiser J, Coward RJ, Inward CD, Farren T, Xing CY, Ni L, Mathieson PW, Mundel P: A conditionally immortalized human podocyte cell line demonstrating nephrin and podocin expression. J Am Soc Nephrol 2002, 13:630-638

21. Jenkins RH, Davies LC, Taylor PR, Akiyama H, Cumbers B, Beltrami C, Carrington CP, Phillips AO, Bowen T, Fraser DJ: miR-192 induces $\mathrm{G}_{2} / \mathrm{M}$ growth arrest in aristolochic acid nephropathy. Am $\mathrm{J}$ Pathol 2014, 184:996-1009

22. Midgley AC, Bowen T, Phillips AO, Steadman R: MicroRNA-7 inhibition rescues loss of epidermal growth factor receptor hyaluronandependent differentiation in fibroblasts. Aging Cell 2014, 13:235-244

23. Zhang SD, Gant TW: Effect of pooling samples on the efficiency of comparative studies using microarrays. Bioinformatics 2005, 21: $4378-4383$

24. Kendziorski C, Irizarry RA, Chen KS, Haag JD, Gould MN: On the utility of pooling biological samples in microarray experiments. Proc Natl Acad Sci U S A 2005, 102:4252-4257

25. Robin X, Turck N, Hainard A, Tiberti N, Lisacek F, Sanchez JC, Müller M: pROC: an open-source package for $\mathrm{R}$ and $\mathrm{S}+$ to analyze and compare ROC curves. BMC Bioinformatics 2011, 12:77

26. Prowle JR, Calzavacca P, Licari E, Ligabo EV, Echeverri JE, Bagshaw SM, Haase-Fielitz A, Haase M, Ostland V, Noiri E, Westerman M, Devarajan P, Bellomo R: Combination of biomarkers for diagnosis of acute kidney injury after cardiopulmonary bypass. Ren Fail 2015, 37:408-416

27. Schley G, Köberle C, Manuilova E, Rutz S, Forster F, Weyand M, Formentini I, Kientsch-Engel R, Kai-Eckardt U, Willam C: Comparison of plasma and urine biomarker performance in acute kidney injury. PLoS One 2015, 10:e145042

28. Navarro J, Milena F, Mora C, Leon C, Claverie F, Flores C, Garcia J: Tumor necrosis factor-alpha gene expression in diabetic nephropathy: relationship with urinary albumin excretion and effect of angiotensin-converting enzyme inhibition. Kidney Int Suppl 2005, 99:S98-S102

29. Ohga S, Shikata K, Yozai K, Okada S, Ogawa D, Usui H, Wada J, Shikata Y, Makino H: Thiazolidinedione ameliorates renal injury in experimental diabetic rats through anti-inflammatory effects mediated by inhibition of NF-кB activation. Am J Physiol Renal Physiol 2007, 292:F1141-F1150 
30. Smith DA, Newbury LJ, Drago G, Bowen T, Redman JE: Electrochemical detection of urinary microRNAs via sulfonamide-bound antisense hybridisation. Sens Actuators B Chem 2017, 253: 335-341

31. Olivieri F, Spazzafumo L, Bonafè M, Recchioni R, Prattichizzo F, Marcheselli M, Micolucci L, Mensà E, Giuliani A, Santini G, Gobbi M, Lazzarini R, Boemi M, Testa R, Antonicelli R, Antonio Domenico Procopio AD, Bonfigli AR: miR-21-5p and miR-126a-3p levels in plasma and circulating angiogenic cells: relationship with type 2 diabetes complications. Oncotarget 2015, 6:35372-35382

32. Barutta F, Bruno G, Matullo G, Chaturvedi N, Grimaldi S, Schalkwijk C, Stehouwer CD, Fuller JH, Gruden G: MicroRNA-126 and micro/macrovascular complications of type 1 diabetes in the EURODIAB prospective complications study. Acta Diabetol 2017, 54:133-139

33. Al-Kafaji G, Al-Mahroos G, Al-Muhtaresh HA, Skrypnyk C, Sabry MA, Ramadan AR: Decreased expression of circulating microRNA-126 in patients with type 2 diabetic nephropathy: a potential blood-based biomarker. Exp Ther Med 2016, 12:815-822

34. Osipova J, Fischer D-C, Dangwal S, Volkmann I, Widera C, Schwarz K, Lorenzen JM, Schreiver C, Jacoby U, Heimhalt M, Thum T, Haffner D: Diabetes-associated MicroRNAs in pediatric patients with type 1 diabetes mellitus: a cross-sectional cohort study. J Clin Endocrinol Metab 2014, 99:E1661-E1665

35. Wang S, Aurora AB, Johnson BA, Qi X, McAnally J, Hill JA, Richardson JA, Bassel-Duby R, Olson EN: The endothelial-specific microRNA miR-126 governs vascular integrity and angiogneneiss. Dev Cell 2008, 15:261-271

36. Brosius FC, Coward RJ: Podocytes, signaling pathways, and vascular factors in diabetic kidney disease. Adv Chronic Kidney Dis 2014, 21: 304-310

37. Harris TA, Yamakuchi M, Ferlito M, Mendell JT, Lowenstein CJ: MicroRNA-126 regulates endothelial expression of vascular cell adhesion molecule 1. Proc Natl Acad Sci U S A 2008, 105:1516-1521

38. Ásgeirsdóttir SA, van Solingen C, Kurniati NF, Zwiers PJ, Heeringa P, van Meurs M, Satchell SC, Saleem MA, Mathieson PW, Banas B, Kamps JA, Rabelink TJ, van Zonneveld AJ, Molema G: MicroRNA126 contributes to renal microvascular heterogeneity of VCAM-1 protein expression in acute inflammation. Am J Physiol Renal Physiol 2012, 302:F1630-F1639

39. Huang Y, Liu Y, Li L, Su B, Yang L, Fan W, Yin Q, Chen L, Cui T, Zhang J, Lu Y, Cheng J, Fu P, Liu F: Involvement of inflammation-related
miR-155 and miR-146a in diabetic nephropathy: implications for glomerular endothelial injury. BMC Nephrol 2014, 15:142

40. Lin X, You Y, Wang J, Qin Y, Huang P, Yang F: MicroRNA-155 deficiency promotes nephrin acetylation and attenuates renal damage in hyperglycemia-induced nephropathy. Inflammation 2015, 38: $546-554$

41. Welten SMJ, Goossens EAC, Quax PHA, Nossent AY: The multifactorial nature of microRNAs in vascular remodeling. Cardiovasc Res 2016, 110:6-22

42. Wang B, Komers R, Carew R, Winbanks CE, Xu B, HermanEdelstein M, Koh P, Thomas M, Jandeleit-Dahm K, Gregorevic P, Cooper ME, Kantharidis P: Suppression of microRNA-29 expression by TGF- $\beta 1$ promotes collagen expression and renal fibrosis. J Am Soc Nephrol 2012, 23:252-265

43. Chen H-Y, Zhong X, Huang XR, Meng X-M, You Y, Chung ACK, Lan HY: MicroRNA-29b inhibits diabetic nephropathy in db/db mice. Mol Ther 2014, 22:842-853

44. Toyono T, Usui T, Villarreal G, Kallay L, Matthaei M, Vianna LMM, Zhu AY, Kuroda M, Amano S, Jun AS: MicroRNA-29b overexpression decreases extracellular matrix mRNA and protein production in human corneal endothelial cells. Cornea 2016, 35:1466-1470

45. Zhu HQ, Li Q, Dong LY, Zhou Q, Wang H, Wang Y: MicroRNA-29b promotes high-fat diet-stimulated endothelial permeability and apoptosis in apoE knock-out mice by down-regulating MT1 expression. Int J Cardiol 2014, 176:764-770

46. Silambarasan M, Tan JR, Karolina DS, Armugam A, Kaur C, Jeyaseelan K: MicroRNAs in hyperglycemia induced endothelial cell dysfunction. Int J Mol Sci 2016, 17:518

47. Mocharla P, Briand S, Giannotti G, Dörries C, Jakob P, Paneni F, Lüscher T, Landmesser U: AngiomiR-126 expression and secretion from circulating $\mathrm{CD} 34^{+}$and $\mathrm{CD} 14^{+}$PBMCs: role for proangiogenic effects and alterations in type 2 diabetics. Blood 2013, 121: 226-236

48. Barutta F, Tricarico M, Corbelli A, Annaratone L, Pinach S, Grimaldi S, Bruno G, Cimino D, Taverna D, Deregibus MC, Rastaldi MP, Perin PC, Gruden G: Urinary exosomal microRNAs in incipient diabetic nephropathy. PLoS One 2013, 8:e73798

49. Salama A, Fichou N, Allard M, Dubreil L, De Beaurepaire L, Viel A, Jégou D, Bösch S, Bach J-M: MicroRNA-29b modulates innate and antigen-specific immune responses in mouse models of autoimmunity. PLoS One 2014, 9:e106153 